\title{
LA SUSTENTABILIDAD OCEÁNICA A TRAVÉS DE LA FIGURA DE ÁNGELES ALVARIÑ̃ ${ }^{1}$
}

\author{
Sabela Domínguez López²
}

\section{La sustentabilidad oceánica a través de la figura de Ángeles Alvariño}

Resumen: A través de la figura de la oceanógrafa Ángeles Alvariño, como modelo de científica que llegó a ser reconocida dentro de la ciencia, a pesar de los obstáculos vividos durante su carrera profesional, se realiza el diseño de un proyecto con perspectiva de género abordando el aprendizaje a través de cuestiones socio-científicas, pretendiendo hacer consciente al alumnado de las dificultades e incomprensión que sufrieron, y sufren, las mujeres en este ámbito y reforzar la autoestima de las alumnas en el proceso de aprendizaje. Dichos obstáculos quedan patentes en un texto adjunto que servirá como recurso al alumnado.

Palabras clave: Ángeles Alvariño, oceanógrafas, proyecto educativo, perspectiva de género, coeducación, sustentabilidad, discriminación de género.

\section{Oceanic Sustainability through the Figure of Ángeles Alvariño}

Abstract: This article approaches the figure of oceanographer Ángeles Alvariño as a scientific role model in order to design a project with a gender perspective that tackles learning through socio-scientific questions, with the aim of improving students awareness of the difficulties and incomprehension that women suffered, and still suffer, in this field and to reinforce students' selfesteem in the learning process. These obstacles are shown in an accompanying text, which serves as an educational resource for students.

Key words: Ángeles Alvariño, women oceanographers, educational project, gender perspective, coeducation, sustainability, gender discrimination.

\section{Introducción}

Como trabajo de fin de Máster (TFM en adelante) del Máster de Formación de Profesorado en Educación Secundaria en la especialidad de Tecnología diseñé el proyecto educativo que presento en este artículo. La decisión de realizarlo responde a la necesidad de implementar la perspectiva de género en materias de ciencia y tecnología, al detectar

\footnotetext{
${ }^{1}$ Fecha de recepción: 28/11/2019.

${ }^{2}$ Investigadora colaboradora del Departamento de Ciencias Experimentales de la Universidad de Vigo, España; $₫$ sabela.domínguez@gmail.com.
} 
una carencia de la misma en el currículum de esta última materia en Secundaria. Así mismo, en una salida didáctica a o Grove y a la Isla de Cortegada que realicé con el alumnado del 3er curso de la ESO del IES Val do Asma (en Chantada - Lugo) quedó patente su interés por el mar. Surge así la idea de trabajarlo a través de la aportación de las mujeres a la oceanografía.

Cuando mi tutora, María Mercedes Álvarez Lires me habló de la oceanógrafa Ángeles Alvariño, me centré en buscar información sobre ella y otras colegas de profesión. Durante mi investigación obtuve información relevante sobre los obstáculos con los que se encontraron estas científicas por el mero hecho de ser mujeres. A partir de esta, se crean dos textos como recursos para trabajar con el alumnado que se adjuntan en el artículo, al ser de gran interés e imprescindibles a la hora de desarrollar este proyecto.

Por falta de tiempo durante el período de prácticas, solamente se pudo realizar la búsqueda del centro de interés mencionada anteriormente, pero posteriormente se llevó a cabo una adaptación de este proyecto en la asignatura de "Educación Ambiental para el Desarrollo" del Grado de Educación Primaria, de donde se dedujeron las conclusiones finales.

\section{Fundamentación}

Los estudios de Ciencia, Tecnología y Género documentan, entre otros aspectos, la ausencia y presencia de la mujer en la historia del desarrollo científico-tecnológico para explicar el porqué de esta situación y proponen estrategias educativas que permitan una incorporación más plena de la mujer en estos campos (González y Pérez-Sedeño 2002: 
1). Autoras como Álvarez-Lires, Nuño, y Solsona (2003) muestran la influencia del androcentrismo en la construcción y producción de la ciencia. Al tener un único enfoque, masculino, se olvida del ámbito de lo privado, ocultando los logros de las mujeres, los saberes relacionados con sus labores tradicionales (como la agricultura, los colorantes, la alimentación, etc.), y en consecuencia, las tecnologías propias de estas, siendo todo lo femenino, invisible para la historia de la ciencia y la tecnología. Otra consecuencia derivada de esta mirada androcentrista es la consideración de la ciencia como una actividad objetiva, lógica y neutral, poseedora de verdades absolutas e incuestionables descubiertas por hombres blancos de clase media-alta. Teniendo en cuenta la concepción dualista del pensamiento occidental, esta perspectiva influye en la consideración positiva de lo masculino frente a lo femenino (como por ejemplo, objetivo vs subjetivo, verdad vs error, racional vs sentimental, fuerte vs débil) (Álvarez-Lires et al 2003: 22).

En el ámbito educativo, si nos fijamos en los contenidos del currículum y en el tratamiento de la información en los libros de texto y materiales didácticos, vemos que están orientados a los intereses masculinos, mayoritariamente (Álvarez-Lires, Nuño et Solsona 2003: 16; González y Pérez-Sedeño 2002: 12); además, la concepción de ciencia desde la perspectiva androcentrista, dificulta el acceso de las mujeres a determinadas profesiones (Álvarez-Lires, Nuño et Solsona 2003: 14; González y Pérez-Sedeño 2002: 6) e incluso, potencia actitudes y comportamientos sociales que favorecen la intolerancia ante opiniones diferentes (Álvarez-Lires et al 2003: 15). Asimismo, el sesgo masculino en la actividad científica no afecta solo a las mujeres, sino también a los hombres y a la propia ciencia (Álvarez-Lires et al 2003: 15; ETAN 2000: 43; González et al 2002: 14; She figures 2003: 73, 2012: 16, 2015: 175).

Por esta razón, diversas autoras manifiestan que se deben realizar cambios en distintos niveles dentro del ámbito educativo (Álvarez-Lires et al 2003: 19) y para esto es 
necesario tomar diversas medidas (Álvarez-Lires et al 2003: 21), entre ellas destacamos las que forman parte de este proyecto. En primer lugar, hacer visible la presencia de las mujeres en la historia de la ciencia, mostrando los obstáculos y la incomprensión que sufrieron. En segundo lugar, tener presentes las experiencias previas de las chicas, ya que existen diferentes interesas, habilidades, aptitudes y actitudes entre los chicos y las chicas. Y por último, cambiar esa visión sexista de la ciencia hacia un enfoque coeducativo, mostrando la ciencia como una construcción personal y social, resaltando la responsabilidad de las comunidades científicas ante los problemas de la humanidad, ya que la ciencia es una construcción humana, y por lo tanto, social.

\section{Objetivos del proyecto}

Teniendo en cuenta el mar, como interés detectado durante una salida didáctica al Grove y a la Isla de Cortegada, y después de toda la reflexión anterior, se proponen como objetivos generales de este proyecto: 1) Abordar el aprendizaje a través de cuestiones sociocientíficas. 2) Potenciar la autoestima de las chicas mediante la utilización de modelos de científicas y actividades orientadas a sus intereses, aptitudes y actitudes. 3) Fomentar en el alumnado el interés y conocimiento del mar, mostrando la importancia de su sustentabilidad. 


\section{Investigación previa}

El proyecto surge de la realización de una visita guiada de Guimatur (Asociación de mariscadoras de Cambados) a la que asistía alumnado de secundaria, en la que se muestran las costumbres costeras y el modo de trabajar de las mariscadoras. De esta visita surge la primera actividad de aplicación.

Acudo al Archivo Municipal de A Coruña, para documentarme sobre la discriminación que sufrió Ángeles Alvariño. De esta investigación surge el documento de la actividad de estructuración y síntesis de la primera tarea, tras consultar las signaturas 2, 47, 111, 114 e 137 del fondo de Ángeles Alvariño. Visito el Instituto Oceanográfico Español (IEO, en adelante), de Vigo, donde obtengo información sobre Ángeles Alvariño y me informan de que el investigador Francisco Rodríguez Hernández, tiene un blog sobre fitoplancton: www.fitopasion.com, del que obtengo recursos sobre plancton, que utilizo en las actividades de estructuración y síntesis de la segunda tarea. Acudo a las jornadas de puertas abiertas del IEO de A Coruña, donde me informan de que realizan talleres para institutos. De esta visita surge la segunda actividad de aplicación.

\section{Diseño y desarrollo del proyecto}

El proyecto que se va a desarrollar lleva por título: La sustentabilidad oceánica a través de la figura de Ángeles Alvariño. Este proyecto se diseña inicialmente para la materia de Tecnología del alumnado del 3er curso de la ESO, aunque se llevó a cabo una adaptación del mismo en la asignatura de Educación Ambiental para el Desarrollo del 3er 
curso del Grado de Educación Primaria de la Universidad de Vigo. También podría hacerse uso de ella en otras materias como pueden ser Biología o Cultura Científica de $4^{\circ}$ curso de la ESO. Para su realización utilizamos la metodología propuesta en el libro de Azucena Arias et al (2009) y para estructurar las actividades y tareas dentro del proyecto, la adaptación de Fanny Angulo (2003) del ciclo de Karplus.

\subsection{Fase introductoria}

En primer lugar, en la fase introductoria del proyecto, se le propone al alumnado como objetivo la realización de un cómic relacionado con el mar, tras observar sus inquietudes sobre este en la salida didáctica mencionada anteriormente. Para realizar el cómic, se necesita una temática, buscar información e interpretarla, para desenvolver la narrativa y la ilustración.

Al ser la materia de tecnología y trabajar la sustentabilidad, tenemos que relacionar la temática con la actividad humana, proponiéndole al alumnado que piense en profesiones relacionadas con el mar. Para esto, utilizaremos la estrategia “1, 2, 4". En primer lugar, cada estudiante piensa en dos profesiones, a continuación, se juntan en parejas y escogen dos de sus cuatro opciones y finalmente se junta el grupo completo, formado por 4 integrantes, quedándose con dos opciones. Se hará una puesta en común anotando en el encerado las respuestas de cada grupo. En este caso, la idea es encaminar al alumnado hacia las oceanógrafas, por lo que si no saliera el término, lo aportaríamos mediante la pregunta: ¿Y qué sabemos de las oceanógrafas? ¿Podrían ellas aportarnos información sobre el mar? 
La temática que se desenvuelve en este proyecto es la sustentabilidad oceánica a través de la figura de Ángeles Alvariño. Utilizamos esta figura como modelo de científica que llegó a ser reconocida centro de la ciencia a pesar de las dificultades con las que se encontró, con lo que pretendemos hacer consciente al alumnado de las dificultades que sufrieron las mujeres en este ámbito y reforzar la autoestima de las alumnas en el proceso de aprendizaje, entre otras cosas, como se mencionó anteriormente. Por otro lado, se observa que la presencia del mar en el currículum escolar es escasa, en consecuencia, el alumnado tiene mucho desconocimiento de este. Por esta razón, me parece fundamental mostrar la importancia de la sustentabilidad oceánica y la labor que realizan las oceanógrafas/os al respecto para que sean conscientes de que su conservación nos afecta a todas las personas.

5.2. Fase de desarrollo

En el transcurso del proyecto, cuyo centro de interés es Ángeles Alvariño, se presentan cuatro posibles tareas a desarrollar, con sus correspondientes actividades y evaluaciones.

\subsubsection{Primera tarea}

Comenzamos el Ciclo de Karplus con la actividad de exploración inicial "veo, pienso, me pregunto". En esta actividad enseñamos una imagen de Ángeles Alvariño y 
cada estudiante responderá en una hoja a las preguntas: ¿Qué veo en la imagen?, ¿qué interpretación hago de la imagen? (con los motivos de su interpretación) y ¿qué me sugiere la imagen? (qué preguntas se hacen y por qué). A continuación, se hará una puesta en común, donde iremos escribiendo en el encerado las ideas de todo el grupo. De esta manera, podemos saber cuáles son las ideas que tienen con respecto a esta persona y sus inquietudes.

Al ver que existen muchas preguntas al respecto y distintas interpretaciones, se propondrá realizar una biografía para saber quién es Ángeles Alvariño. Para determinar qué información adicional necesitamos para hacer una biografía utilizamos la estrategia del folio giratorio en grupo. Anotamos en el encerado las diferentes aportaciones en la puesta en común, completando así el ¿qué queremos saber? El siguiente paso es organizar la información recopilada en ambas estrategias. En este caso, se organizan cuatro grupos de estudiantes con cuatro integrantes cada uno, distribuyendo la información de la siguiente manera: vida personal, estudios e intereses, vida laboral y trabajos a los que se dedicó, y reconocimientos y destrezas.

Ahora que ya sabemos ¿Qué queremos saber?, nos preguntamos: ¿Dónde buscamos? Para responder a esta pregunta, se les ofrecerá una serie de recursos: http://marenfeminino.campusdomar.gal/?page_id=189: Web con una breve biografía y un enlace al libro de Alberto González-Garcés Santiso: Ángeles Alvariño, investigadora marina de relevancia mundial; https://mujeresconciencia.com/2015/06/12/angelesalvarino-cientifica-gallega-2015/: Web con enlaces a varias biografías, en diversos formatos: vídeo, díptico y narrativa en: "Más información"; https://oceanicas.ieo.es/angeles-alvarino-gonzalez-la-fuerza-de-un-caracter/: Web de la FECYT sobre las oceanógrafas con biografía de Alvariño. Del mismo mod, se les 
informará de la existencia del libro: Un ronsel de Ardora, que podrán obtener de la biblioteca, de estudiantes, amistades, etc.

Para realizar la búsqueda de información, nos preguntamos: ¿Cómo nos organizamos? Para esta actividad de introducción de conocimientos, utilizamos la estrategia cooperativa del "grupo de expertas y expertos". En esta estrategia, a los grupos iniciales de cuatro estudiantes los llamamos grupos base. A cada integrante del grupo base se le asigna un número del 1 al 4, para repartir de un modo ordenado la distinta información que tenemos que buscar: El número 1: Vida personal, 2: Estudios e intereses, 3: Vida laboral e investigaciones, 4: Reconocimientos y destrezas.

Una vez recogida la información, se hacen nuevos grupos formados por estudiantes con experiencia en el mismo tema, o lo que es lo mismo, con el mismo número, con el objetivo de contrastar la información y aportar información que les haya parecido importante para la resolución de las preguntas. A continuación, se vuelve al grupo base para poner en común toda la información recopilada por cada miembro. Así, todos los miembros del grupo conocerán todos los puntos a tocar en la biografía en interacción con el resto de estudiantes. Finalmente, se anotan en el encerado todos los términos difíciles de entender y se intentan resolver conjuntamente con la/el docente, pudiendo iniciarse una nueva actividad de investigación.

Como actividad de estructuración y síntesis, cada grupo base ordenará la información en una línea de tiempo de la vida de Ángeles Alvariño. La siguiente actividad de introducción de conocimientos pretende que el alumnado reflexione sobre el contexto en el que vivió Alvariño y el resto de oceanógrafas para que elaboren razonamientos de manera crítica sobre su vida, como mujer e investigadora. Para esto, se utilizan dos textos que se encuentran en el apartado 6: el primero habla sobre las oceanógrafas precursoras 
en España y el segundo sobre la discriminación de género que vivió Alvariño en la institución SouthWest Fisheries Center (SWFC).

Utilizarán la estrategia de la lectura compartida para leer los textos. Dos integrantes del grupo leerán uno de los textos y la otra pareja, el otro, desarrollándose de la siguiente manera: el primer párrafo del texto es leído por una persona en voz alta, la otra escucha de forma activa y al terminar la lectura hace un resumen. La persona que leyó el texto decide si la explicación es correcta o no, y una vez que llegan a consenso, anotan la explicación. En el siguiente párrafo se intercambian los papeles y así sucesivamente, hasta que terminen el texto. Una vez leídos los dos textos, cada pareja, explicará a la otra de qué trata el texto que leyó.

Ahora que toda la clase sabe la información de ambos textos, utilizamos la estrategia del debate para hacer una puesta en común, realizando esta de una manera ordenada, esperando el turno de palabra, dando un razonamiento de la idea expuesta y promoviendo el respeto ante las ideas del resto. Como actividad de estructuración y síntesis realizarán una reflexión individual sobre las sensaciones que les producen, su opinión y razonamientos con respecto al trabajo de estas científicas y los obstáculos que se les presentaron, después de investigar la trayectoria profesional de Alvariño y que opinan de los logros y los obstáculos que se les presentaron a estas científicas.

La actividad de aplicación será realizar la biografía de Ángeles Alvariño en grupo base. Como ejemplo, se les dará la biografía de Rachel Carson, para que les sirva de guion. La biografía será expuesta por cada grupo con la ayuda visual de un PowerPoint, contribuyendo a la exposición todos los miembros el equipo. Los grupos restantes realizarán preguntas al grupo expositor y si existen preguntas sin resolver, se anotan para 
su posterior resolución. El resto de grupos evaluarán la exposición mediante una tabla de evaluación.

Finalmente, realizaremos una coevaluación respondiendo a las preguntas: ¿Qué hemos aprendido? Haremos una puesta en común para que cada estudiante sea consciente de su aprendizaje. ¿Y qué queremos aprender? Igualmente, se hará una puesta en común de lo que al alumnado le interesa para llevar a cabo el proyecto. Como dentro de la oceanografía Ángeles Alvariño destacó como planctóloga, se considera la posibilidad de que el plancton sea una de las respuestas, generándose una nueva investigación sobre el plancton, que corresponderá a la siguiente tarea.

\subsubsection{Segunda tarea}

Comenzamos un nuevo ciclo de Karplus relacionado con el plancton. Como actividad de exploración utilizaremos la estrategia de la plaza del pueblo, desenvolviéndose de la siguiente manera: primeramente, se le proporciona a cada estudiante un cuestionario al que se responderá cada pregunta con un Sí o un No. Cada pregunta tendrá que ser respondida por una persona distinta. Para levar esto a cabo, el grupo de estudiantes se distribuye por la clase en busca de personas que respondan a sus preguntas, hasta completar el cuestionario.

Una vez completo, la/el docente comienza a preguntar la respuesta de cada pregunta de manera ordenada: se le pregunta a un/a estudiante la respuesta que tiene a la primera pregunta y quién se la respondió. A continuación, se le pregunta a la persona que respondió el motivo de su respuesta. Una vez que da su razonamiento, se le pregunta qué 
respuesta tiene anotada a esa misma pregunta y quién se la respondió, para que nos de su explicación también. Se pide el motivo de por qué pusieron que sí o no que no hasta que tengamos una conclusión de que tenemos seguridad de cuál es la respuesta correcta.

Tabla I. Cuestionario para la estrategia "La plaza del pueblo"

\begin{tabular}{|c|c|c|c|}
\hline Pregunta & Sí & No & Nombre \\
\hline $\begin{array}{l}\text { 1. Se llama plancton a los organismos que se encuentran en la superficie } \\
\text { del agua }\end{array}$ & & & \\
\hline 2. Las larvas de los peces forman parte del plancton & & & \\
\hline 3. Las mareas rojas son proliferaciones de algas & & & \\
\hline 4. El plancton se alimenta de microalgas & & & \\
\hline 5. El mar de ardora se produce con la combustión del plancton & & & \\
\hline 6. El fitoplancton siempre permanece en la superficie del agua & & & \\
\hline 7. El plancton mide menos de $1 \mathrm{~mm}$ & & & \\
\hline 8. Las almejas son depredadoras de plancton & & & \\
\hline 9. Las medusas son un tipo de plancton & & & \\
\hline 10. Los crustáceos forman parte del plancton & & & \\
\hline 11. Las ballenas se alimentan de plancton & & & \\
\hline 12. El plancton realiza la fotosíntesis & & & \\
\hline 13. El petróleo y el gas natural se forman a partir del plancton & & & \\
\hline 14. Los dinoflagelados son algas macroscópicas & & & \\
\hline $\begin{array}{l}\text { 15. Rocas, como la caliza, se forman por la compactación de esqueletos de } \\
\text { seres vivos como el plancton. }\end{array}$ & & & \\
\hline 16. Las redes tipo bongo y las nasas se utilizan para recoger plancton & & & \\
\hline $\begin{array}{l}\text { 17. Las mareas rojas son un problema económico para la industria } \\
\text { pesquera porque mueren muchos bivalvos por su causa. }\end{array}$ & & & \\
\hline
\end{tabular}

Se realiza el procedimiento anterior con todas las cuestiones y se irán anotando en el encerado los distintos razonamientos del alumnado a cada una de las preguntas, para 
deducir qué sabemos y qué queremos saber. Una vez finalizada la estrategia, sabemos que información de la que aparece en el cuestionario está clara y cual no, de tal forma que ya hicimos una explicación de ideas previas, y al mismo tiempo, el alumnado es consciente de sus contradicciones y dudas.

A partir de esta información, hacemos un guion de lo que queremos saber para responder a esas dudas.

1. ¿Qué es el plancton? (1)

2. ¿Cuánto mide el plancton? Ejemplos explicativos. (7)

3. ¿Por qué es importante el plancton? $(12,13,15)$

4. ¿Qué tipos de plancton existen? Ejemplos explicativos. $(2,9,10,14)$

5. ¿Cuál es el hábitat del plancton? (6)

6. ¿De qué se alimenta el plancton? $(4,11,12)$

7. ¿Qué se alimenta de él? $(8,11)$

8. ¿Qué quiere decir bioluminiscente? ¿El plancton lo es? Ejemplos explicativos. (4)

9. ¿Qué es una marea roja? $(3,17)$

10. ¿Qué es el mar de ardora? (5)

11. ¿Qué instrumentos se utilizan en la investigación del plancton? (16)

¿Dónde buscamos^? Para buscar la información se aportan los siguientes recursos: https://www.britannica.com/science/plankton : Artículo sobre el plancton con actualizaciones de diferentes autoras y autores que forman parte de instituciones 
relacionadas con la biología marina; http://planktonchronicles.org/es/episodios/: Vídeos del proyecto "Crónicas del plancton" realizado por la Expedición Tara océanos y el Observatorio Oceanológico de Villefranche-sur-mer, donde muestran distintas variedades de plancton con explicaciones de las distintas clases (algunos en castellano y otros en inglés); https://oceanexplorer.noaa.gov/edu/themes/bioluminescence/ welcome.html: Web con varios artículos hablando sobre la bioluminiscencia, donde pueden investigar qué es, qué especies la producen, los motivos que desencadenan que la produzcan y de qué manera se produce; https://www.youtube.com/watch?time_continue $=8 \& v=23 m r t G C k A H 8$ (4”30”“): Vídeo "Say thanks Plankton” filmado por el "Centre National de la Recherche Scientifique" (CNRS) donde muestran cinco razones por las que tenemos que darle gracias al plancton: por el aire que respiramos, por la comida que nos proporciona, por calentar nuestra casa y movernos en los coches, por las construcciones que se hacen con rocas generadas por ellos y por nuestra propia existencia y la del resto de especies. Sacado do blog de Francisco Rodríguez Hernández, investigador do Instituto Español de Oceanografía (IEO): https://fitopasion.com/; y Vídeo 2: https://www.mareasvermellasgalicia.com/ (22"24”“"): Documental sobre las mareas rojas donde se explica que son producidas por la proliferación de fitoplancton, pudiendo ser estas tóxicas, afectando a la explotación de los moluscos bivalvos de la costa gallega, y como consecuencia a la economía costera, en https://www.mareasvermellasgalicia.com/. De igual modo, se les recomendará para las dudas con el inglés el diccionario y la aplicación de linguee: https://www.linguee.es/ y el traductor deepl: https://www.deepl.com/translator.

¿Cómo nos organizamos? Para esta actividad de estructuración y síntesis, utilizaremos la estrategia del intercambio entre equipos. Consiste en la búsqueda de información de dos o tres subtemas distintos por cada grupo: Grupo 1: Qué es el plancton, 
cuanto mide y la importancia del plancton. Grupo 2: Tipos de plancton, hábitat e instrumentos de investigación. Grupo 3: alimentación y depredadores. Grupo 4: bioluminiscencia y que son las mareas rojas.

Una vez recogida la información, se selecciona, se contrasta, se entiende y esquematiza, para posteriormente hacer el intercambio de equipos y comunicarla. A continuación, se hacen nuevos grupos compuestos por un integrante de cada equipo base, para esto, se le asigna un número del 1 al 4 a cada estudiante dentro del grupo base y los nuevos grupos estarán formados por estudiantes del mismo número. Una vez hechos los nuevos grupos, se intercambia la información. Finalmente, los estudiantes vuelven al grupo base y ponen en común toda la información.

Realizarán dos actividades de estructuración y síntesis: un mapa mental sobre el plancton, para ordenar la información recogida, contrastada y resumida y una rueda de aprendizaje, que pretende generar una síntesis de la información importante. Esta última estrategia consiste en responder a las siguientes cuestiones relacionadas con lo que piensa el alumnado sobre el tema que aprendió: ¿qué es lo más importante para mí?, ¿qué no debería olvidar?, ¿qué es lo que más me impactó? y ¿qué es lo más interesante para mí? De esta manera, el alumnado es más consciente de su aprendizaje y de lo que le parece más importante o no sabía, siendo la manera de recordar y estructurar lo aprendido. Después de esta actividad, realizaremos de nuevo una coevaluación del proceso a través de las respuestas a las preguntas: ¿qué hemos aprendido? ¿y qué queremos aprender? 


\subsubsection{Tercera tarea}

Se propone una salida didáctica como actividad tanto de introducción de conocimientos, como de aplicación. Sería un ejemplo de solapamiento entre dos fases, ya que estarán en contacto con personas expertas que resolverán sus dudas y aplicarán lo aprendido anteriormente al compartir el trabajo de personas expertas. Esta actividad, a su vez, se divide en dos actividades:

En la primera, conoceremos las labores de las mujeres relacionadas con el mar. Los bivalvos se alimentan de plancton y ya vieron anteriormente que alguna proliferación de plancton puede ser tóxica, afectando a los bivalvos y en consecuencia a la economía costera. Se pretende proporcionar un contacto con la realidad para que el alumnado comience a darle importancia tanto a la sustentabilidad oceánica como a los trabajos de las mujeres. En esta harán una visita guiada con Guimatur, la Asociación Cultural "Mujeres del Mar" de Cambados, conociendo la cultura y el estilo de vida de la gente dedicada al mar. A mayores, colaborarán con el colectivo de mariscadoras, explicándoles el proceso del marisqueo.

En esta experiencia aprenderán sobre la captura de las especies, diferencias entre ellas, sus depredadores, las herramientas que se utilizan, como se organizan, la colaboración y el compañerismo entre ellas, los requisitos para ser mariscadora, los controles que pasan, los distintos trabajos que realizan a parte de la extracción de moluscos de talla comercial: limpiezas de las playas, preparación del terreno para sembrar, las resiembras, vigilancias, etc., haciendo su trabajo sustentable y ayudando a la conservación de los océanos. 
En la segunda actividad, iremos al IEO de A Coruña, donde el alumnado realizará una actividad divulgativa diseñada por personal investigador del IEO. En esta, les explicarán las áreas principales de estudio del instituto: Área de pesquerías, Área de medio marino y protección ambiental y Área de acuicultura, explicando el funcionamiento de diversos aparatos de muestreo en el mar. Además darán una charla sobre biodiversidad, y la importancia del plancton en el medio marino y la cadena trófica, que terminará con la observación de muestras de fitoplancton y zooplancton en un analizador de imagen.

El alumnado debe de recoger datos que considere relevante durante ambas actividades, e incluso sacar fotografías o realizar bocetos, ya que tendrán que aplicar lo aprendido en el cómic. Se hará una puesta en común de las dudas resueltas por cada grupo y de las conclusiones y relaciones que observamos en las actividades realizadas. Nuevamente utilizaremos la estrategia de las cuatro flechas como actividad de aplicación y evaluación (en este caso, se realizará un cuadro por cada actividad realizada) y realizaremos una coevaluación del proceso de aprendizaje (en este caso, damos por hecho que el alumnado solucionó todas sus dudas y se siente seguro para realizar la actividad final).

\subsubsection{Cuarta tarea}

Como actividad de aplicación final, realizarán un cómic, que era el objetivo inicial del proyecto. Cada grupo base, realizará su propio cómic valiéndose del material generado durante el proyecto. Ángeles Alvariño, el plancton y las mariscadoras. 
Aparecerán todos los temas tocados en el cómic, siendo la protagonista principal Ángeles Alvariño. Como recurso, pueden consultar cómics de la biblioteca para observar diferentes estilos.

5.2.5. Fase de valoración o comunicación

Todos los grupos harán una exposición de los cómics en la clase, explicando su temática y desarrollo. En la exposición participarán todos los miembros de cada grupo y será evaluada también por el resto estudiantes del aula mediante una tabla de evaluación. Tras la exposición el resto de grupos, podrán hacer preguntas sobre lo expuesto. Una vez hecha esta evaluación presentarán el fruto de su proyecto al mundo para que este lo valore, de manera que el que obtenga la mayor votación, será publicado en la revista del centro. El alumnado escogerá la vía por la que se hará esta votación.

Finalmente, procedemos a hacer la evaluación del proyecto por el alumnado. Se realizará mediante un cuestionario con kahoot! que tendrán que responder, de forma anónima: al nivel de dificultad de las sesiones, valoración de la utilidad de las estrategias para la comprensión del tema, y un autoanálisis: la importancia que le da a la temática, la comunicación, la búsqueda de información y al aprendizaje. 


\section{Recursos de interés}

Con la información obtenida durante la investigación previa al proyecto se realizan los siguientes textos con los que trabajará el alumnado. Con ellos se pretende dar a conocer las dificultades con las que se encontró esta científica y el resto de oceanógrafas.

6.1. Pioneras que contribuyeron a la incorporación de la mujer a la oceanografía en España

Luisa de la Vega Wetter Y Josefa Sanz Echeverría fueron las precursoras de la oceanografía en España. Luisa, de origen francés, comenzó a trabajar en la Estación Marítima de Zoología y Botánica experimental de Santander, que funda su marido Augusto González de Linares en 1886. Allí estuvo reproduciendo en acuarela los ejemplares más importantes de la fauna marina de la zona hasta que falleció su marido, momento en el que se encaminó a la docencia (Madariaga 2004: 75). La segunda mujer en incorporarse a la estación fue Josefa, que, a pesar de no tener estudios formales como naturalista, poseía gran destreza en la recolección, preparación y disección de animales marinos, por influencia de su tío. Esta destreza le ayudó a convertirse en una excepcional ictióloga, de hecho, a pesar de que acabo trabajando en el Museo de Ciencias Naturales, la llamaban para colaborar ocasionalmente en el análisis de muestras de las campañas oceanográficas (Feigl y Barreiro 2005: 3).

El Instituto Español de Oceanografía (IEO en adelante) fue creado en 1914 con la intención de centrarse íntegramente en la investigación del mar y sus recursos. La primera 
mujer en incorporarse a esta institución fue Jimena Quirós en 1921, que junto a Emma Bardán, $M^{a}$ Encarnación Sánchez y $\mathbf{M}^{\mathrm{a}}$ de las Mercedes García formaron la primera generación de oceanógrafas de esta institución. Entre los logros de estas científicas, Quirós fue pionera en publicar un artículo en la revista del IEO (Feigl 2005: 1) y en embarcar en un buque oceanográfico (Lozano 2018) y Bardán fue la primera investigadora marina española en asistir y presentar sus resultados en un congreso internacional en el ICES en 1949 (Feigl 2005: 1). Estos avances se vieron truncados por la guerra Civil y la dictadura de Franco, ya que después de 1939 las investigadoras de esta institución no pudieron embarcar en buques de investigación hasta los años 70 (González-Garcés 2016: 26). Tras la postguerra, en 1940 a Quirós la cesaron de su puesto por haber pertenecido al Partido Radical Socialista y haber recibido diferentes cargos dentro de este partido -participó en varios mítines como ponente, defendió el sufragio femenino y lideró la sección femenina del partido en 1932- (Lozano 2018). Cuando instalaron la sede del IEO en Madrid, el resto de pioneras fueron destinadas allí para realizar labores de coordinación y gestión, dejando de publicar desde entonces. Sin embargo, algunos autores reconocieron la colaboración anónima de Bardán en sus obras. Esta última, además de haber sido la primera oceanógrafa en asistir y presentar sus resultados en un congreso internacional, publicó 10 artículos sobre aspectos pesqueros de Canarias y Málaga antes de su traslado a Madrid (Feigl 2005: 1).

La segunda generación de oceanógrafas corresponde a la época de la post-guerra, cuando se consolidó el acceso de las mujeres al IEO, de 1941 a 1951. Esta generación estuvo formada por 8 mujeres, siendo las más representativas $M^{a}$ Dolores García Pineda, doctora en Farmacia y Bioquímica y Ángeles Alvariño (Feigl 2005: 2). A partir de entonces, hasta 1969 no se admitió a ninguna nueva autora (Feigl y Barreiro 2005: 6). 
La representante más destacada de la $3^{\text {a }}$ generación es Josefina Castellví. Esta, en los años 60 se convirtió en la primera oceanógrafa del CSIC (Consejo Superior de Investigaciones Científicas) en el ICM (Instituto de Ciencias Marinas). Realizo 36 campañas oceanográficas y fue la primera española que investigó la Antártida (Feigl y Barreiro 2005: 6).

6.2. Vivencias de Ángeles Alvariño en la SouthWest Fisheries Center

Después de una larga trayectoria como investigadora en el mundo de la pesca y la oceanografía, Ángeles Alvariño empieza a trabajar en la SouthWest Fisheries Center (SWFC), en la Jolla, California. Ingresa en esta institución con un nivel intermedio a pesar de corresponderle un nivel superior debido a su experiencia; ella acepta igualmente bajo la condición de promocionar en el momento que exista alguna vacante en un puesto superior.

A Alvariño, con el tiempo, le sorprende y le desilusiona ver como sus colegas hombres, con menos experiencia y muchos, con currículos inferiores al suyo (como los recién salidos de la universidad), son promovidos y ella continúa en el mismo puesto a pesar de la promesa que le hicieron y una larga experiencia a la espalda. Así que cada vez que publican una vacante, intenta un aumento de su status, aunque este siempre es denegado por sus superiores.

En el sistema de promociones existente en aquel momento en esta institución, una persona no era valorada por sus méritos y reconocimiento, sino por el trabajo que desenvolvía. Este trabajo era definido inicialmente por el supervisor, la gerencia lo 
aprobaba y dependiendo de las funciones y responsabilidades desempeñadas en este, determinaban el nivel del empleado. Esta era una de las razones que le daban a Alvariño para denegarle el ascenso, su superior, Smith y su subdirector, Barrett: que sus funciones y responsabilidades, definidas por ellos mismos, no eran evaluables para una promoción,

Su relación con su superior Smith no era buena, no existía comunicación. Alvariño consideraba que Smith retrasaba su trabajo y Smith juzgaba la arrogancia de Alvariño. En una ocasión, Alvariño informa al director de la SWFC de que se siente maltratada como profesional por Smith. Expone que este, se dirige a ella como una "clasificadora de plancton", le hace peticiones para el manuscrito de Sagitta Scrippsae que él mismo sabe que no pueden ser cumplidas y la culpa, haciendo cargos insensatos relacionados con la producción y el profesionalismo de su trabajo.

Ante las negativas de sus superiores, Alvariño pide que se reconsidere su promoción por razones para ella, obvias, pero Smith se lo pone difícil. Se esfuerza en disminuir sus funciones en el laboratorio por miedo a la posible repercusión que podría ocasionar la promoción de la oceanógrafa. Opinaba que los hombres del laboratorio se sentirían desmoralizados por no tener el entrenamiento y la formación de Alvariño, y que esto afectaría a la productividad y al sentido de realización personal de sus compañeros.

En la SWFC existía la comisión EEO (Equal Employment Opportunity) destinada a "combatir" cualquier tipo de discriminación. Cada cierto tiempo miembros de la comisión acudían a la SWFC para comprobar el status de las minorías y de las mujeres. Alvariño tuvo varias entrevistas con ellos pero nunca fueron fructíferas. Durante un año, fue representante de las mujeres empleadas en el SWFC y realizó estudios estadísticos de la situación de la mujer. Observó con respecto a las mujeres, que ella era la única con un puesto 12 y las demás se encontraban por debajo del 9. Dedujo como consecuencia de 
esto, que no existían mujeres ni en puestos de supervisión, ni en las comisiones de toma de decisiones o funciones similares, ni en las comisiones de recomendación de ascensos y reclamó un cambio de esta situación para que existiese realmente una igualdad de oportunidades en el empleo.

Ante tal situación, Alvariño se siente constantemente frustrada al encontrarse con impedimentos para prosperar en la SWFC. Le niegan acudir a cursos diseñados para gerentes, supervisores y ascensos (a los que siempre acuden los mismos), la asistencia a seminarios, simposios y congresos, e incluso la tutela de estudiantes de post-doctorado y doctorado. Para más inri, descubre en los archivos de su información personal que han modificado datos, cambiando las fechas de sus títulos académicos y sus calificaciones, que siendo de matrícula, aparecen en hojas en blanco anexas al documento modificado.

Después de siete años, ya cansada de esta situación, Alvariño escucha en la NBC a Juanita Kreeps, Secretaria del Departamento de Comercio de los Estados Unidos, hablando de la discriminación de género en el trabajo. Se ve reflejada en sus palabras y decide escribirle a ella y al presidente de los EEUU, comentándoles la información de sus estudios de género dentro de la institución y la experiencia que vivió y teme vivir, como le pasó otras veces, si la carta cae en manos de "los zorros que cuidan de sus pollos". En esta carta habla también de la persecución personal que a la que se ha visto sometida, de discriminación racial, nepotismo y falta de tratamiento humanista a los empleados en la SWFC. Considera a esta institución una mafia donde le resulta imposible superarse porque "los hombres tienen el control" y "hacen el esfuerzo por acumular en el lugar a los de su propia especie".

Durante años (desde 1971 a 1978), Alvariño luchó por una promoción que nunca llegaba ya que acabó jubilándose en el mismo nivel en el que comenzó 17 años antes. El 
logro de esa lucha fue un aumento de su salario a costa de recurrir las negativas y teniendo que testificar en una presentación oral, donde la resolución le fue favorable. Se habla de Alvariño como una mujer de carácter fuerte y arrogante, defensora de sus ideas. Tenemos que agradecer que esas características formasen parte de su personalidad, ya que si no fuera así, puede que nunca hubiese llegado a ser reconocida. Debemos pensar, que parte de ese carácter fue forjado por las vivencias que experimentó a lo largo de su vida como mujer, luchando por reivindicar su profesionalidad y feminidad. De hecho, puede que si en vida le hubiesen dado el reconocimiento merecido, no se hubiese visto obligada a reafirmar tan a menudo sus méritos. ${ }^{3}$

\section{Conclusiones}

Como comenté en la introducción, aunque este proyecto fue diseñado para la materia de Tecnología del curso de $3^{\circ}$ de la ESO, no fue posible su desarrollo por falta de tiempo, por lo tanto, fue necesario realizar una adaptación posterior de las actividades para su aplicación en la asignatura de Educación Ambiental para el Desarrollo del Grado de Educación Primaria de la Universidad de Vigo.

Al no poder realizar la salida didáctica de la tarea 3, por un lado, nos convertimos en "oceanógrafas/os" en el laboratorio, observando al microscopio, dibujando y analizando muestras de plancton y por otro, utilizamos un vídeo de la presidenta de la

\footnotetext{
${ }^{3}$ La información del texto se extrae del fondo de Ángeles Alvariño que se encuentra en el Archivo Municipal de A Coruña. Signaturas: 2, 47, 111, 114 e 137.
} 
asociación de mariscadoras a pie Guimatur, para conocer su historia, manera de trabajar y de hacer sustentable su trabajo.

Las conclusiones que se pueden deducir del cuestionario final es que el uso de figuras de científicas y de los obstáculos e incomprensión que sufrieron en su carrera profesional, nos ayuda a visualizarlas, a generar empatía hacia ellas y su modo de afrontar dichas dificultades, a interesarnos por su trabajo y a valorarlo, tanto por parte del alumnado como del profesorado. Así mismo, en algunos momentos nos identificamos con su lucha mostrando una mayor admiración por la oceanógrafa. Por otro lado, mostrar el trabajo que realizan las mariscadoras sirvió para tomar conciencia de la labor que desempeñan y empoderarlas. Por otra parte, aprender sobre el mar a partir de cuestiones socio-científicas ayudó al alumnado a interesarse por este tema y a tomar conciencia sobre la importancia de contribuir a su conservación. Con respecto a las estrategias cooperativas utilizadas, en las que prima el diálogo y el respeto, quedó patente su aceptación por parte de las alumnas.

\section{Referencias bibliográficas}

Álvarez Lires, María Mercedes, Nuño Angós, Teresa y Solsona Pairó, Nuria (eds.) 2003. Las científicas y su historia en el aula. Madrid: Síntesis.

Angulo, F, García-Rovira, M. P. 2003. Un modelo didáctico para la Formación Inicial del Profesorado de Ciencias. Intercomunitaria de Formación del Profesorado 17.1. 3749.

Archivo Municipal de A Coruña (AMC). Fondo Ángeles Alvariño. Signatura 2: "Memorándum sobre la resolución negativa a petición del aumento de salario".

Archivo Municipal de A Coruña (AMC). Fondo Ángeles Alvariño. Signatura 111: "Material relativo a la discusión verbal por la negativa de ascenso de categoría y aumento de salario. Recensiones bibliográficas para su publicación en boletines y 
revistas, currículum y papeles personales de trabajo, donde se reflejan los problemas con los que se encontró a lo largo de su carrera".

Archivo Municipal de A Coruña (AMC). Fondo Ángeles Alvariño. Signatura 47: "Borradores de cartas denunciando la situación de trabajo en la SWFC, dependiente de la National Oceanic and Atmospheric Administration (NOAA)".

Archivo Municipal de A Coruña (AMC). Fondo Ángeles Alvariño. Signatura 114: "Documentación sobre asuntos relacionados con su cualificación laboral. Discriminación por sexo y raza, las condiciones de trabajo en la SWFC y más documentación sobre la seguridad en el trabajo".

Archivo Municipal de A Coruña (AMC). Fondo Ángeles Alvariño. Signatura 137: "Vida laboral y puesto de trabajo. Comunicación administrativa para responder y arreglar impresiones y cargos erróneos de P. E. Smith de 1973. Datos sobre discriminación del 1974. Colaboración y asesoramiento del Dr. W. F. Royce en 1974”.

Arias Correa, A.; Arias Correa, D.; Navaza Blanco, M.V., e Rial Fernández, M.D. 2009. $O$ traballo por proxectos, en infantil, primaria y secundaria. Santiago de Compostela: Xunta de Galicia. Consellería de Educación y Ordenación Universitaria.

ETAN. 2000. "European Teachnology Assessment Network on Women and Science. Expert Working Group on Women and Science. Science Policies in the European Union. European Commission, Research Directorate general”. Brusels.

European Commission. 2003. She Figures 2003, Women and Science Statistics and Indicadors. Brussels: Office for Official Publications of the European Communities.

European Commission. 2012. She Figures 2012, Women and Science Statistics and Indicadors. Brussels: Office for Official Publications of the European Communities.

European Commission. 2015. She Figures 2015, Women and Science Statistics and Indicadors. Brussels: Office for Official Publications of the European Communities.

Feigl, Juan Pérez-Rubín. 2005. "Pioneras en la investigación oceanográfica y pesquera en el IEO”. Instituto Español de Oceanografía 1: 14-16.

Feigl, Juan Pérez-Rubín. 2015. "La oceanógrafa Ángeles Alvariño (1916-2005): Desde Galicia para el mundo". Real Academia Galega de Ciencias 34: 5-60.

Feigl, Juan Pérez-Rubín; Wulff Barreiro, Enrique. 2005. "Women pioneers of marine and freshwater sciences in Spain: IEO (1923-1969) and CSIC (1943-1969)". IX Congreso de la Sociedad Española de Historia de las Ciencias y de las Técnicas (SEHCYT). Eds. Francisco J. González, Juan A. Pérez-Bustamante, José C. Martín, Enrique Wulff, José F. Casanueva y Francisco Herrera. Cádiz: SEHCYT 2: 10971107.

Feigl, Juan Pérez-Rubín. y Wulff Barreiro, Enrique. 2011. “Ángeles Alvariño (19162005), the pioneering Spanish female oceanographer with the largest international 
exposure". X Congreso de la Sociedad Española de Historia de las Ciencias y de las Técnicas (SEHCYT). Eds. José M. Cobos Bueno, Antonio Pulgarin Guerrero y Elena Ausejo. Badajoz: SEHCYT. 255-268

González-Garcés Santiso, Alberto. 2016. Ángeles Alvariño González, investigadora marina de relevancia mundial. Instituto Español de Oceanografía. Madrid: Ministerio de Economía y Competitividad.

González García, Marta I. y Pérez-Sedeño, Eulalia. 2002. “Ciencia, tecnología y género”. Revista iberoamericana de Ciencia, Tecnología, Sociedad e Innovación 2: 5.

Lozano, Pablo. 2018. “Jimena Quirós: la Guerra Civil truncó la carrera de la primera oceanógrafa en la historia de España". Oceánicas. https://oceanicas.ieo.es/jimenaquiros-la-primera-oceanografa-en-la-historia-de-espana-cuya-carrera-trunco-laguerra/. [04/12/2018].

Madariaga, Benito. 2004. Augusto González de Linares. Vida y obra de un naturalista. Santander: Instituto Español de Oceanografía. 